\title{
The effect of inertia, viscous damping, temperature and normal stress on chaotic behaviour of the rate and state friction model
}

\author{
Nitish Sinha ${ }^{1}$, Arun K Singh ${ }^{1, *}$ and Trilok N Singh ${ }^{2}$ \\ ${ }^{1}$ Department of Mechanical Engineering, Visvesvaraya National Institute of Technology, Nagpur 440 010, India. \\ ${ }^{2}$ Department of Earth Sciences, Indian Institute of Technology Bombay, Mumbai 400 076, India. \\ *Corresponding author.e-mail: aksinghb@gmail.com
}

MS received 14 March 2017; revised 10 September 2017; accepted 12 September 2017; published online 16 April 2018

A fundamental understanding of frictional sliding at rock surfaces is of practical importance for nucleation and propagation of earthquakes and rock slope stability. We investigate numerically the effect of different physical parameters such as inertia, viscous damping, temperature and normal stress on the chaotic behaviour of the two state variables rate and state friction (2sRSF) model. In general, a slight variation in any of inertia, viscous damping, temperature and effective normal stress reduces the chaotic behaviour of the sliding system. However, the present study has shown the appearance of chaos for the specific values of normal stress before it disappears again as the normal stress varies further. It is also observed that magnitude of system stiffness at which chaotic motion occurs, is less than the corresponding value of critical stiffness determined by using the linear stability analysis. These results explain the practical observation why chaotic nucleation of an earthquake is a rare phenomenon as reported in literature.

Keywords. Rate and state friction; inertia; viscous damping; temperature; normal stress; chaotic motion of rock sliding.

\begin{tabular}{|c|c|c|c|}
\hline \multicolumn{2}{|c|}{ List of notations and abbreviations } & $c$ & $\begin{array}{l}\left(1-\beta_{1} q_{1}\right) Q_{a} / R T^{*}+\left(1-\beta_{2} q_{2}\right) Q_{a} / R T^{*} \\
\rho=L_{1} / L_{2}\end{array}$ \\
\hline$\tau$ & Dimensional frictional stress $\left(\mathrm{P}_{\mathrm{a}}\right)$ & $K$ & Stiffness of connecting spring $\left(\mathrm{P}_{\mathrm{a}} \mathrm{m}^{-1}\right)$ \\
\hline$\tau^{*}$ & Reference frictional stress $\left(\mathrm{P}_{\mathrm{a}}\right)$ & $k$ & $K L_{1} / \sigma_{n} a$, non-dimensional spring stiff- \\
\hline$\sigma_{n}$ & Effective normal stress $\left(\mathrm{P}_{\mathrm{a}}\right)$ & & ness \\
\hline$\mu_{*}$ & Reference coefficient of friction & $r$ & Ratio of inertial time to frictional charac- \\
\hline$\theta_{1}, \theta_{2}$ & $\begin{array}{l}\text { State variable related to asperity contact } \\
\text { of the sliding interface }\end{array}$ & & \\
\hline$a, b_{1}$, & Constants related to rate and state fric- & $\hat{r}$ & $\sqrt{m v_{*}^{2}} / \sigma_{n} a L_{1}$ \\
\hline & tion & $\hat{\gamma}$ & $\gamma v_{*} / \sigma_{n} a$ \\
\hline$Q_{a}, Q_{b_{1}}$ & $\begin{array}{l}\text { Activation energies corresponding to } a, b_{1} \\
\text { and } b_{2}\end{array}$ & $\hat{\gamma}$ & $\begin{array}{l}\text { Nondimensional viscous damping coeffi- } \\
\text { cient }\end{array}$ \\
\hline $\begin{array}{l}Q_{b_{2}} \\
c_{1}\end{array}$ & Nondimensional term relates to frictional & $m$ & Mass of the sliding block $(\mathrm{kg})$ \\
\hline & heating & $\beta_{1}$ & $b_{1} / a$ \\
\hline$c_{2}$ & Nondimensional term relates to heat con- & $\beta_{2}$ & $b_{2} / a$ \\
\hline & duction & & \\
\hline
\end{tabular}




$\begin{array}{ll}q_{2} & Q_{b_{2}} / Q_{a} \\ \psi & \tau / \sigma a \\ \phi & \ln \left(v / v_{*}\right) \\ d_{c} & \text { Critical slip distance }(\mathrm{m}) \\ v_{0} & \text { Pulling velocity }\left(\mathrm{ms}^{-1}\right) \\ R & \text { Universal gas constant }\left(\mathrm{J} \mathrm{K}^{-1} \mathrm{~mol}^{-1}\right) \\ T_{*} & \text { Reference temperature }(\mathrm{K}) \\ T & t v_{*} / L_{1} \\ T_{s} & \text { Temperature of the sliding interface }(\mathrm{K}) \\ \hat{T}_{s} & T_{s} / T_{*}\end{array}$

\section{Introduction}

In recent years, theoretical and experimental studies have established that friction along the earthquake faults plays a critical role in controlling their stability (Brace and Byerlee 1966; Dieterich 1979; Rice and Ruina 1983; Carlson and Langer 1989; Marone 1998). The rate and state friction (RSF) model is widely used to understand the dynamics of the earthquake process, for example, nucleation of earthquake, post-seismic relaxation process, etc. (Rice and Ruina 1983; Singh and Singh 2013; Rice and Tse 1986; Marone 1998). In recent times, the RSF model is also used for studying rock slope stability (Chau 1995; Helmstetter et al. 2004; Faillettaz et al. 2010; Singh et al. 2012). The RSF model is basically an extension of the classical Amontons-Coulombs (ACs) laws (Marone 1998). Based on the friction experiments at rock surfaces, Dieterich (1979), Ruina (1983) and Rice and Ruina (1983) proposed the RSF constitutive laws. Accordingly, frictional stress $\tau$ of rock surfaces depends on slip velocity $v$ and the state variable $\theta$ for a constant normal stress $\sigma_{n}$, which is generally expressed as:

$$
\tau=\sigma_{n}\left[\mu_{*}+a \ln \frac{v}{v_{*}}+b \ln \frac{v_{*} \theta}{L}\right]
$$

where $\theta$ is a state variable signifying the history of the sliding surfaces (Ruina 1983). Further, $a$ and $b$ are the constants related to the 'direct effect' that is, increase in shear stress with slip velocity, while the 'evolution effect' causes gradual decrease in shear resistance of asperity contacts, respectively. The material constant $L$ signifies the characteristic slip distance over which evolution of friction occurs due to direct effect (Ruina 1983). Furthermore, $\mu_{*}$ and $v_{*}$ are reference frictional shear stress and shear velocity corresponding to the AC laws, respectively. Two basic laws have originally been proposed for the 'state variable' $\theta$ namely the Ruina-Rice slip law/slip law $d \theta / d t=-v \theta \ln (v \theta / L) / L$ and the other law is known as Dieterich-Ruina aging law/aging law, that is, $d \theta / d t=1-\theta v / L$ (Ruina 1983; Marone 1998; Ranjith and Rice 1999). Under the steady sliding conditions, both friction laws result in the same expression for steady dynamic stress as well as critical stiffness (Ruina 1983; Ranjith and Rice 1999). However, a basic difference between these two laws is that, unlike the slip law, the aging law results in true time of stationary contact (Ruina 1983; Ranjith and Rice 1999). It has also been shown numerically that the RSF with the slip law results in chaos, but the same is not seen with the aging law (Liu 2007; Sinha and Singh 2016a,b). The reason for this contradicting behaviour is not known in literature (Liu 2007). Thus, further study is needed to unfold the mystery behind this issue.

Chaos is defined as 'Aperiodic long-term behaviour in a deterministic system that exhibits sensitive dependence on initial conditions (SICs)' (Strogatz 1994). Although chaos is a general manifestation of a non-linear dynamical system, it is not observed in all non-linear systems (Strogatz 1994). A necessary condition for chaos of a continuous dynamical system is that the solutions of the system of differential equations must show the dependency on SICs (Strogatz 1994; Lakshmanan and Rajasekar 2003). In addition, an important condition for the chaos of a such dynamical system is that it must possess at least three independent variables coupled with at least one non-linear term (Strogatz 1994; Lakshmanan and Rajasekar 2003). For instance, although the RSF with one state variable rate and state friction (1sRSF) is often used to explain stiffness dependence of stick-slip instability under both quasistatic and inertial conditions, 1 sRSF does not result in chaotic motion (Ruina 1983; Rice and Ruina 1983; Gu et al. 1984; Marone 1998). However, the addition of one more state variable in the $1 \mathrm{sRSF}$ model results in the chaotic motion and this is known as the two state variables based rate and state dependent friction (2sRSF) model (Ruina 1983; Gu et al. 1984). Thus, equation (1) gets modified owing to two state variables $\theta_{1}$ and $\theta_{2}$ as following:

$$
\tau=\sigma_{n}\left[\mu_{*}+a \ln \frac{v}{v_{*}}+b_{1} \ln \frac{v_{*} \theta_{1}}{L_{1}}+b_{2} \ln \frac{v_{*} \theta_{2}}{L_{2}}\right] .
$$


Similarly, both the state variable laws also get modified as $d \theta_{i} / d t=-v \theta_{i} \ln \left(v \theta_{i} / L_{i}\right) / L_{i}$ and $d \theta_{i} / d t=1-\theta_{i} v / L_{i}$, where $i=1,2$. The $2 \mathrm{sRSF}$ was proposed to match the step velocity experiments and has also been reported its chaotic behaviour (Ruina 1983). Gu et al. (1984) have studied numerically the chaotic behaviour of the $2 \mathrm{sRSF}$ model in detail and reported that the $2 \mathrm{sRSF}$ with the slip law indeed results in the chaotic motion via period doubling bifurcation. Rice (1993, 1996) has also discussed the complexity involved during an earthquake with the rate and state friction. $\mathrm{Gu}$ and Wong (1994) used a slightly modified form of the 2sRSF model to study the chaotic behaviour of sliding rock surfaces at higher sliding velocity and they have also confirmed that the period doubling route to chaos with the experiments. Niu and Chen (1994) have also investigated the period doubling behaviour of the $2 \mathrm{sRSF}$ in detail using the different numerical tools for instance, Lyapunov Exponents, fractal dimensions, etc. Becker (2000) concluded through the numerical simulations that irregular behaviour of the $2 \mathrm{sRSF}$ can be reduced to one-dimensional unimodel mapping; thus the route of chaos is period doubling. Liu (2007) compared the linear and non-linear behaviour of the $2 \mathrm{sRSF}$ with the slip law as well as the aging law and reported the chaotic behaviour with slip law, but not with the aging law. Xuejun (2013) has also carried out linear and non-linear stability analysis of the $2 \mathrm{sRSF}$ using the numerical tools such as bifurcation diagram, phase diagram and Lyapunov exponents. Recently, we have also studied the chaotic behaviour of three state variables rate and state dependent friction (3sRSF) and observed that the system becomes more chaotic than the 2sRSF model (Sinha and Singh 2016a, b). Burridge and Knopoff (1967) have proposed a chaotic model for seismicity along the earth fault using the modified multiple spring mass system which is known as Burridge and Knopoff (B-K) model. This promising model has extensively been studied since its proposal. Brittany et al. (2011) have used the RSF to study the chaotic behaviour of the $\mathrm{B}-\mathrm{K}$ model considering both discrete as well as corresponding continuum model. Kawamura et al. (2017) have also done the statistical analysis of the $\mathrm{B}-\mathrm{K}$ model in the light of rate and state friction.

Despite all these numerical studies, it is not known in literature how viscous damping, inertia, normal stress, temperature, etc., related to the RSF affects its chaotic behaviour. In the present article, we have investigated numerically the effect of aforementioned parameters on the chaotic behaviour of the $2 \mathrm{sRSF}$ with the slip law. We have employed the time series and phase plot to discuss the results.

\section{Modelling of governing differential equations}

We use a standard spring-mass slider having mass $m$, viscous damping $\gamma$ and spring stiffness $K$ per unit area of the sliding mass. The free end of the spring is being pulled with a constant velocity $V_{0}$ and the other end of spring is connected to the sliding mass with velocity of center of mass $v(\mathrm{Gu}$ et al. 1984; Ranjith and Rice 1999). The governing equation for the slider is given by

$$
K\left(V_{0}-v\right)-\dot{\tau}-\gamma \dot{v}=m \ddot{v}
$$

and the dimensionless form of equation (3) is obtained

$$
\begin{aligned}
\frac{d^{2} \phi}{d T^{2}}= & r^{-2} e^{-\phi}\left[k\left(v_{0}-e^{\phi}\right)-\frac{d \psi}{d T}-\hat{\gamma} e^{\phi} \frac{d \phi}{d T}\right] \\
& -\left(\frac{d \phi}{d T}\right)^{2} .
\end{aligned}
$$

Further, the non-dimensional form of equation (2) is obtained in terms of equation (5) as:

$$
\psi=\phi+\beta_{1} \hat{\theta}_{1}+\beta_{2} \hat{\theta}_{2}
$$

Also the non-dimensional form of the slip law for both the state variables is obtained as:

$$
\begin{aligned}
& \dot{\hat{\theta}}_{1}=-e^{\phi}\left(\phi+\hat{\theta}_{1}\right), \\
& \dot{\hat{\theta}}_{2}=-\rho e^{\phi}\left(\phi+\hat{\theta}_{2}\right) .
\end{aligned}
$$

In order to derive the system of governing differential equation, we have eliminated $\hat{\theta}_{1}$ and in light of equations (2-6)

$$
\left\{\begin{aligned}
\frac{d^{2} \phi}{d T^{2}}= & r^{-2} e^{-\phi}\left[k\left(v_{0}-e^{\phi}\right)-\dot{\phi}\left(1+\hat{\gamma} e^{\phi}\right)\right. \\
& +e^{\phi}\left\{\left(\beta_{1}-1\right) \phi+\psi-\beta_{2} \hat{\theta}_{2}\right\} \\
& \left.+\rho \beta_{2} e^{\phi}\left(\phi+\hat{\theta}_{2}\right)\right] \\
\frac{d \psi}{d T}= & \dot{\phi}-e^{\phi}\left[\left(\beta_{1}-1\right) \phi+\psi-\beta_{2} \hat{\theta}_{2}\right] \\
& -\rho \beta_{2} e^{\phi}\left(\phi+\hat{\theta}_{2}\right) \\
\frac{d \hat{\theta}_{2}}{d T}= & -\rho e^{\phi}\left(\phi+\hat{\theta}_{2}\right)
\end{aligned}\right.
$$



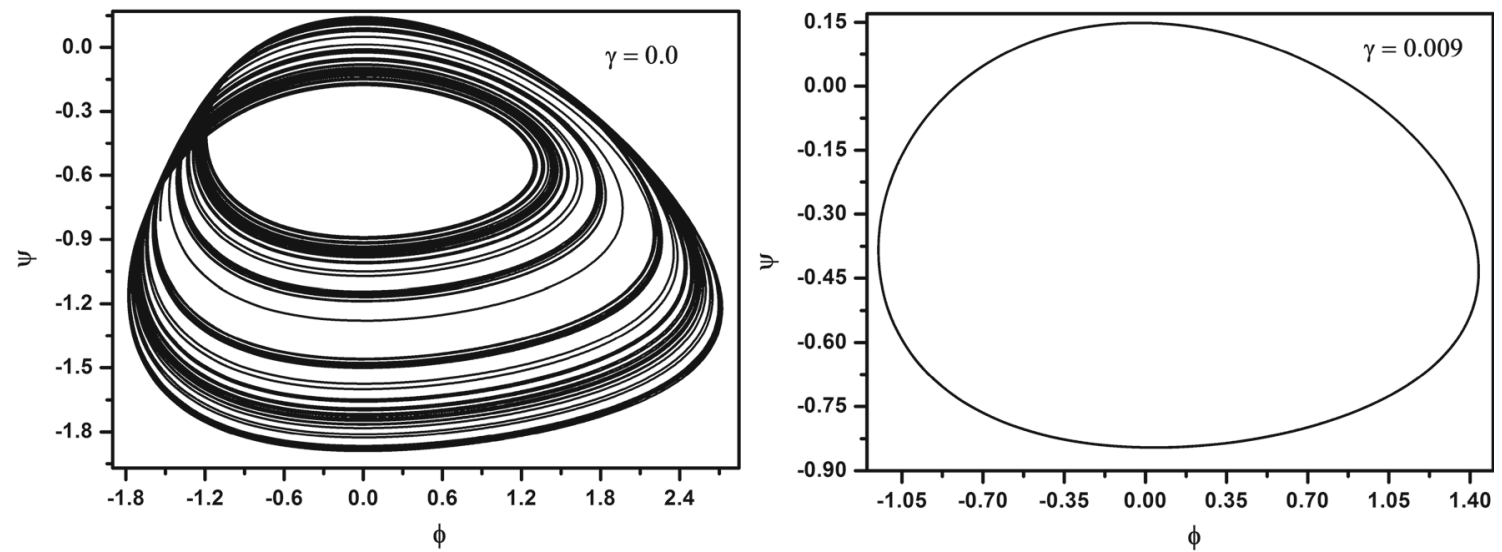

Figure 1. Phase diagrams concerning $\psi$ vs. $\phi$ for viscous damping $\hat{\gamma}=0$ and $\hat{\gamma}=0.009$ at fixed $\beta_{1}=1.0, \beta_{2}=0.84$, $\rho=0.048, k=0.068843$ and initial condition $[0.005,0,0]$.
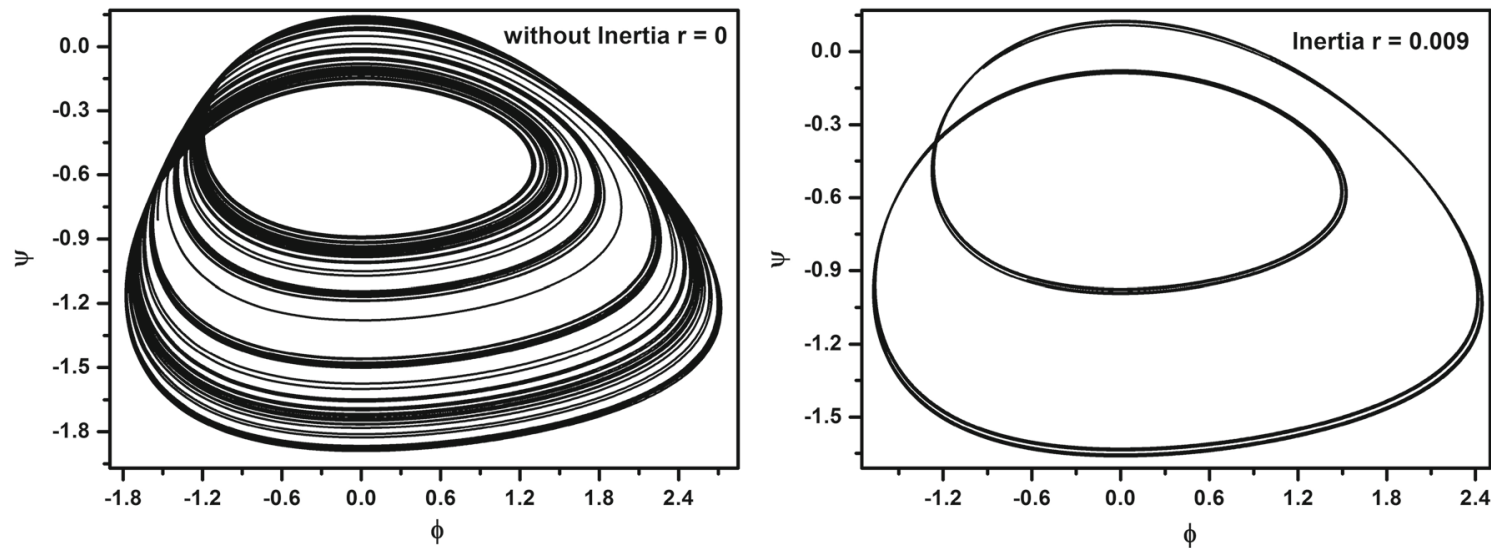

Figure 2. The phase diagrams concerning the effect of inertia $r$ on chaotic behaviour of the 2sRSF model with inertia $(r=0.009)$ and without inertia $(r=0)$ for fixed value of $\beta_{1}=1.0, \beta_{2}=0.84 \rho=0.048$ and $k=0.068843$ at initial condition $[0.005,0,0]$.

In the above derivations from equations (2-7), the dimensionless terms are defined as:

$$
\begin{aligned}
\psi & =\left(\tau-\tau^{*}\right) /\left(\sigma_{n} a\right), \phi=\ln v / v_{*}, \\
\hat{\theta}_{1} & =\ln \left(v_{*} \theta_{1} / L_{1}\right), \hat{\theta}_{2}=\ln \left(v_{*} \theta_{2} / L_{2}\right), \\
T & =v_{*} t / L_{1}, \rho=L_{1} / L_{2}, \\
\beta_{1} & =b_{1} / a, \beta_{2}=b_{2} / a, k=K L_{1} /\left(\sigma_{n} a\right), \\
r^{2} & =m v_{*}^{2} /\left(\sigma_{n} a L_{1}\right), \hat{\gamma}=\gamma v_{*} /\left(\sigma_{n} a\right) .
\end{aligned}
$$

The role of dimensionless inertia and viscous damping are also investigated on chaotic behaviour of the $2 \mathrm{sRSF}$ model, where $r$ is the ratio of inertial time to frictional characteristic time, which is generally expressed as $r^{2}=m v_{*}^{2} /\left(\sigma_{n} a L_{1}\right)$ and viscous damping $\hat{\gamma}=\gamma v_{*} /\left(\sigma_{n} a\right)$ (Ranjith and Rice 1999). It is to be noted that one gets the quasi-static condition of sliding upon neglecting the inertial term $r$ and equation (7) reduces to the original $2 \mathrm{sRSF}$ model (Niu and Chen 1994, 1995; Becker 2000; Xuejun 2013). The 2sRSF friction also becomes
1sRSF law, if one of the state variables is neglected (Ruina 1983; Rice and Ruina 1983). In the next section, we have simulated the system of ordinary differential equation in equation (7) using ode $23 \mathrm{~s}$ solver of MATLAB ${ }^{\circledR}$.

\section{Results and discussion}

\subsection{Effect of viscous damping and inertia on chaotic behaviour of 2sRSF law}

The phase diagrams (figure 1) show that chaotic behaviour of the sliding system reduces to periodic oscillation as magnitude of viscous damping increases from $\hat{\gamma}=0$ to $\hat{\gamma}=0.009$ for a fixed value of spring stiffness $k=0.068843$. This result is expected as viscous damping, in general, eliminates the oscillatory behaviour of a vibrating system (Lakshmanan and Rajasekar 2003). Similarly, the phase diagrams (figure 2) also show that even a small change in inertial term $r$ in equation 
(7) causes chaotic motion to the period doubling. Further increase in $r$ results in complete elimination of the chaos. This result is again consistent with the Duffing system in which a slight increase in mass eliminates its chaotic behaviour (Lakshmanan and Rajasekar 2003). Gu and Wong (1994) have also pointed out that increase in inertia or mass of the sliding system results in period redoubling of the $2 \mathrm{sRSF}$ model.

\subsection{Effect of temperature on chaotic behaviour of the 2sRSF model}

We have also investigated numerically the effect of temperature on chaotic behaviour of the $2 \mathrm{sRSF}$ model. Singh and Singh (2016) studied numerically the linear stability of the rate, state and temperature with one state variable that is, $1 \mathrm{sRSTF}$ model. They have followed the Segall and Rice (2006) approach to model an earthquake fault using the spring-mass system. The numerical simulations of the 1sRSTF have established that it is the relative competition between velocity weakening effect and thermal strengthening effect that controls the frictional stability of the sliding surfaces (Singh and Singh 2016). In this paper, we have derived the governing system of differential equations for two state variables based rate, state and temperature friction that is, 2sRSTF. The governing differential equation of the $2 \mathrm{sRSTF}$ with the spring-mass slider is derived the following the same procedure as discussed in the modelling section of this paper. The final system of governing differential equation is given by

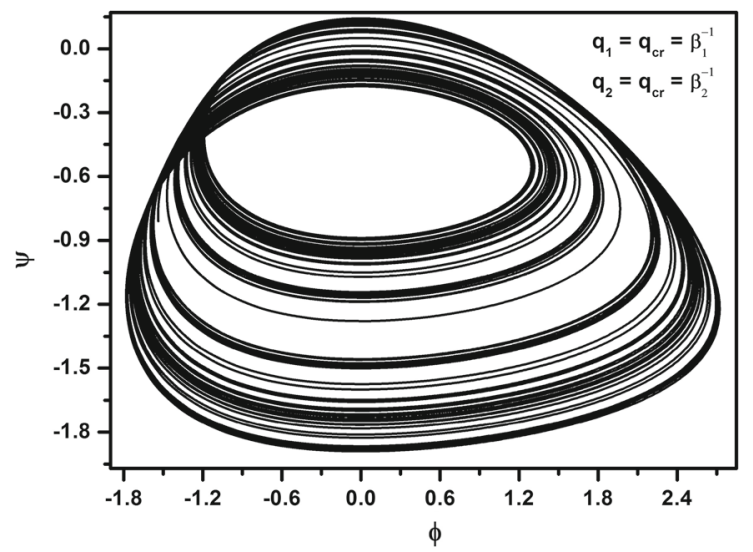

$$
\left\{\begin{aligned}
\frac{d \phi}{d T}= & k\left(v_{0}-e^{\phi}\right)+e^{\phi}\left[\left(\beta_{1}-1\right) \phi+\psi-\hat{\mu}_{*}-\beta_{2} \hat{\theta}_{2}\right. \\
& \left.-c\left(\hat{T}_{s}^{-1}-1\right)\right]+\rho \beta_{2} e^{\phi}\left(\phi+\hat{\theta}_{2}\right) \\
& +c \hat{T}_{s}^{-2}\left[c_{1} e^{\phi} \psi-c_{2}\left(\hat{T}_{s}-\hat{T}_{\mathrm{inf}}\right)\right] \\
\frac{d \psi}{d T}= & k\left(v_{0}-e^{\phi}\right) \\
\frac{d \hat{\theta}_{2}}{d T}= & -\rho e^{\phi}\left(\phi+\hat{\theta}_{2}\right) \\
\frac{d \hat{T}_{s}}{d T}= & c_{1} e^{\phi} \psi-c_{2}\left(\hat{T}_{s}-\hat{T}_{\mathrm{inf}}\right)
\end{aligned}\right.
$$

The non-dimensional terms in equation (8) are defined as $\beta_{1}=b_{1} / a, \beta_{2}=b_{2} / a, \hat{\mu}_{*}=\mu_{*} / a, q_{1}=$ $Q_{b 1} / Q_{a}, q_{2}=Q_{b 2} / Q_{a}$ and $c=Q_{a}\left(1-\beta_{1} q_{1}\right) / R T_{*}+$ $Q_{a}\left(1-\beta_{2} q_{2}\right) / R T_{*}$, where $Q_{a}, Q_{b 1}$ and $Q_{b 2}$ are activation energies corresponding to the physical parameters $a, b_{1}$ and $b_{2}$, respectively. Further, $c_{1}$ and $c_{2}$ are the constants related to heat generation and heat dissipation respectively (Singh and Singh 2016). Equation (8) also includes dimensionless terms namely temperature of the sliding interface $\hat{T}_{s}$ and surrounding temperature $\hat{T}_{\text {inf }}$. It is important to mention that the $2 \mathrm{sRSTF}$ in equation (8) results in the original $2 \mathrm{sRSF}$ for $q_{1}=\left(q_{c r}=\beta_{1}^{-1}\right)$ and $q_{2}=\left(q_{c r}=\beta_{2}^{-1}\right)$.

The numerical simulations of equation (8) reveal that, in general, a slight change in any parameter results in diminishing the chaotic behaviour of the sliding system. For instance, figure 3 shows that as $q_{1}$ increases from $q_{1}=1.00$ (which corresponds to the $2 \mathrm{sRSF}$ model) to $q_{1}=1.005$, the chaotic behaviour reduces to the regular motion. The similar observation is also seen in the case of change in $q_{2}$ for a fixed $q_{1}=1.00$. The reason

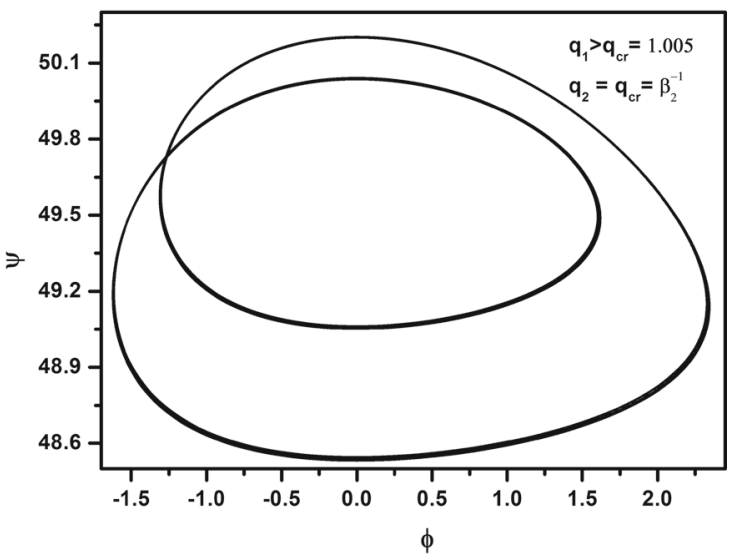

Figure 3. The phase diagrams $(\psi v s . \phi)$ related to the effect of temperature strengthening parameter $q_{1}$ on chaotic behaviour of the $2 \mathrm{sRSF}$ system for $q_{1}=1.0=\left(q_{c r}=\beta_{1}^{-1}\right)$ and $q_{1}=1.01$ but fixed values of $q_{2}=1.1905=\left(q_{c r}=\beta_{2}^{-1}\right), \beta_{1}=1.0$, $\beta_{2}=0.84, \rho=0.048, \mathrm{c}_{1}=1.30 \times 10^{-4}, \mathrm{c}_{2}=2.4 \times 10^{-5}, k=0.068843$ and initial condition $[0.005,50,0,1]$. 

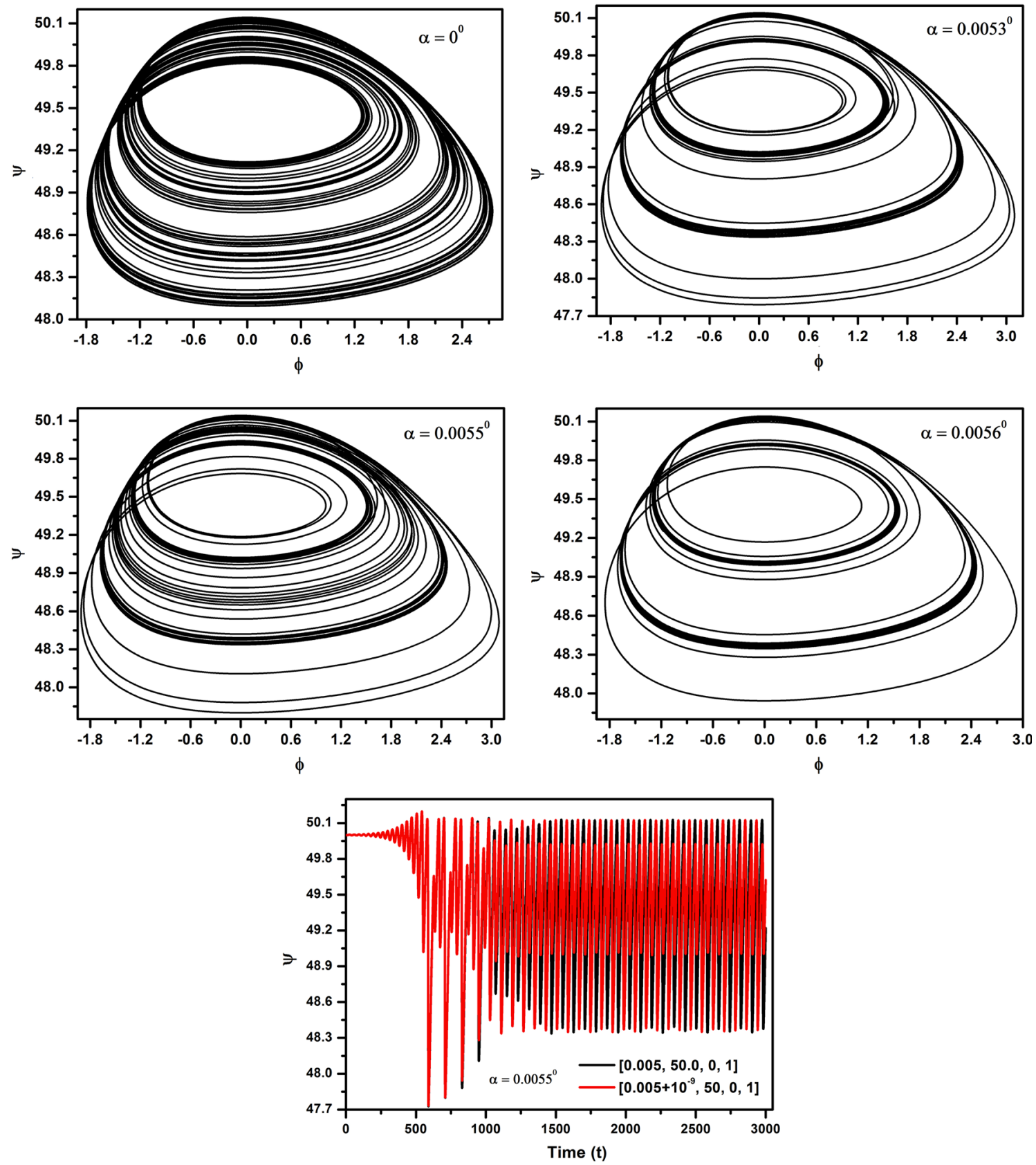

Figure 4. The phase diagrams concerning the chaotic behaviour of the $2 \mathrm{sRSF}$ model for varying $\alpha=0^{0}, \alpha=0.0053^{0}$, $\alpha=0.0055^{\circ}$ and $\alpha=0.0056^{\circ}$ but fixed $\beta_{1}=1.0, \beta_{2}=0.84, \rho=0.048$ and $k=0.068843$ and initial condition $[0.005,50,0,1]$. Time series plot is also presented for $\alpha=0.0055^{\circ}$ for showing stress dependence of sensitive to initial conditions.

for this observation may be attributed to the temperature strengthening effect of $q_{1}$ and $q_{2}$, which reduce the velocity weakening effect thereby the chaotic motion as well. Moreover, other parameters related to the $2 \mathrm{sRSTF}$ such as $c_{1}$ and $c_{2}$ have also been found to be the reducing effect on the chaotic behaviour. These observations are independent of initial temperature $\hat{T}_{s}$ of the sliding surfaces that is, $\hat{T}_{\mathrm{inf}}>\hat{T}_{s}$ or $\hat{T}_{\mathrm{inf}}<\hat{T}_{s}$. It is also concluded that the stiffness value at which irregular motion occurs is less than the critical stiffness obtained (Appendix).

\subsection{Effect of normal stress on chaotic behaviour of the 2sRSF model}

Linker and Dieterich (1992) proposed the role of varying normal stress on frictional dynamics with the RSF model and have also introduced an additional term in the state variable laws to match the 
experimental data. In the present study, we have not considered that additional term to avoid further complexity in the system of the differential equation. Dupont and Bapna (1994) studied theoretically the effect of normal stress on frictional stability with one state variable law. Following the approach of these researchers, we have also derived the governing differential equations for varying normal stress and two state variables which may be stated as the two state variables based rate, state and normal stress friction (2sRSNF) model. The non-dimensional form of the system of governing differential equations is derived as following the same procedure as mentioned earlier

$$
\left\{\begin{aligned}
\frac{d \phi}{d T}= & \hat{\sigma}_{n}^{-1} k\left(v_{0}-e^{\phi}\right)-\hat{\sigma}_{n}^{-2} k\left(v_{0}-e^{\phi}\right) \psi \tan (\alpha) \\
& +\rho \beta_{2} e^{\phi}\left(\phi+\hat{\theta}_{2}\right)+e^{\phi}\left[\left(\beta_{1}-1\right) \phi\right. \\
& \left.-\beta_{2} \hat{\theta}_{2}-\hat{\mu}_{*}+\psi / \hat{\sigma}_{n}\right] \\
\frac{d \psi}{d T}= & k\left(v_{0}-e^{\phi}\right) \\
\frac{d \hat{\theta}_{2}}{d T}= & -\rho e^{\phi}\left(\hat{\theta}_{2}+\phi\right) \\
\frac{d \hat{\sigma}_{n}}{d T}= & -k\left(v_{0}-e^{\phi}\right) \tan (\alpha)
\end{aligned}\right.
$$

where parameter $\alpha$ is basically angular inclination of the connecting spring from the horizontal in the spring-mass sliding system, that acts also like coupling parameter which converts the normal stress into the shear stress (Linker and Dieterich 1992; Dupont and Bapna 1994). Non-dimensional effective normal stress $\hat{\sigma}_{n}$ is defined as $\hat{\sigma}_{n}=\sigma_{n} / \sigma_{*}$, where $\sigma_{*}$ and $\sigma_{n}$ are reference normal stress and fixed normal stress, respectively. While the remaining non-dimensional terms are defined the same as earlier in this article. Noting that similar to equation (8), equation (9) also reduces to the $2 \mathrm{sRSF}$ for $\alpha=0^{0}$.

The numerical simulations of the 2sRSNF model in equation (9) are also presented in figure 4 . It is seen that the chaotic tendency of the sliding system decreases as $\alpha$ increases slightly from $\alpha=0^{0}$ to $\alpha=0.0053^{0}$. However, there is a sudden appearance of chaotic motion at $\alpha=0.0055^{\circ}$ before it disappears again for $\alpha>0.0055^{0}$. The time series plot in figure 4 confirms the evidence of chaos at $\alpha=0.0055^{0}$. For example, it is seen in figure 4 that a slight change in magnitude of $\alpha$ from $\alpha=0.0055^{0}$ to $\alpha=\left(0.0055+10^{-9}\right)^{0}$ results in deviation of stress with time. The possible reason for the appearance of chaos in the $2 \mathrm{sRSNF}$ is due to decrease in adhesive contacts between asperities at the sliding interface. This results in decrease of the effective normal stress and increases in frictional stress for $\alpha>0$. In the case of negative spring angle, adhesive contact among asperities, in contrast, increases because of increase in effective normal stress thus suppressing any irregular motion or chaos at the sliding surfaces (Tolstoi 1967; Cochard et al. 2003). In the case of $2 \mathrm{sRSNF}$, it is again found that critical stiffness $\left(k_{c r}=0.080275\right)$ is larger than the stiffness value $(k=0.068848)$ at which chaos occurs. The details of linear stability of the $2 \mathrm{sRSNF}$ are also described in Appendix.

Present study has shown that in addition to viscous damping, inertia, temperature and normal stress also diminish the chaotic motion of the sliding surfaces. At the same time, the chaotic motion is seen for very specific values of the friction parameters in case of normal stress. On the basis of these results, it is concluded that the chaotic motion may not be always a cause for the nucleation and propagation of an earthquake. This also explains the practical observation why chaotic nucleation and propagation of an earthquake is a rare phenomenon as reported in literature (Huang and Turcotte 1990; Shelly 2010).

\section{Conclusions}

The present numerical simulations establish that viscous damping, inertia, temperature and normal stress, in general, eliminate the chaotic behaviour of the sliding system. However, in case of change in the coupling parameter $\alpha=0.0053^{0}$ to $0.0056^{0}$ related to the normal stress, results in appearance of the chaotic motion for $\alpha=0.0055^{0}$. The critical stiffness $k_{c r}$ obtained with the linear stability analysis of the $2 \mathrm{sRSF}$ model is $k_{c r}=0.088$ which is larger in magnitude than the stiffness $k=0.068843$ at which chaotic motion occurs. It is also concluded that why chaotic motion of the sliding surfaces is a rare phenomenon in crustal earthquakes and failure of the rock slopes. Since most of the parameters of the RSF results in reducing the chaotic effect of the sliding system.

\section{Acknowledgements}

This work is supported by NRDMS-DST (order No. NRDMS//02/43/016(G)). The authors would like to thank Prof. Vinay A Juvekar, IIT Bombay 
for his useful discussion and suggestions for the improvement of the present manuscript.

\section{Appendix}

\section{A1. Linear stability of 2sRSTF model}

In this section, we have carried out linear stability of the 2sRSTF model for predicting the critical stiffness at which sliding behaviour changes from stable to unstable. The system of differential equations in equation (8) is linearized about the equilibrium point or fixed point. This is obtained by equating the time derivatives in equation (8) equal to zero and solving the system of algebraic equations numerically. Moreover, the corresponding Jacobian matrix of equation (8) is obtained as: $s_{0} s_{3}=0$ and $s_{1} s_{2} s_{3}-s_{0} s_{3}^{2}-s_{4} s_{1}^{2}=0$. These two coupled non-linear algebraic equations are, in turn, solved numerically for critical stiffness.

\section{A2. Linear stability of 2sRSNF model}

In this section, we have also carried out linear stability of the $2 \mathrm{sRSNF}$ model for predicting the critical stiffness of the system. We followed the same procedure for evaluating critical stiffness of the $2 \mathrm{sRSTF}$ friction model as is done in the case of 2 sRSTF. The system of equilibrium point of equation (9) is obtained about the steady sliding as $\phi_{s s}=e^{v_{0}}, \hat{\theta}_{2 s s}=-\phi_{s s}, \hat{\sigma}_{n s s}=1$ and $\psi_{s s}=\hat{\sigma}_{n s s}\left[\hat{\mu}_{*}+\left(1-\beta_{1}-\beta_{2}\right) \phi_{s s}\right]$. This is now used to obtain the Jacobian matrix as following:

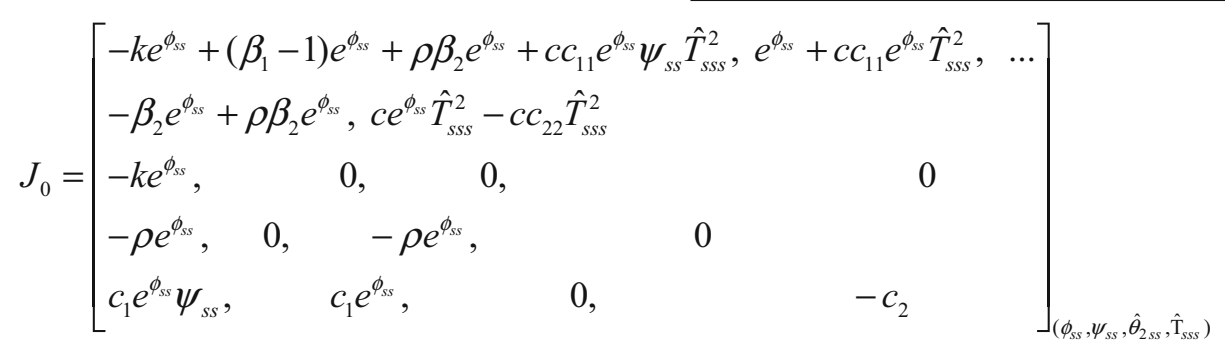

The characteristic polynomial is obtained by expanding the above Jacobian matrix and this is given by $s_{0} \lambda^{4}+s_{1} \lambda^{3}+s_{2} \lambda^{2}+s_{3} \lambda+s_{4}=0$, where the co-efficient of the polynomial are expressed as:

$$
\begin{aligned}
s_{0}= & 16, \\
s_{1}= & 8\left(c_{2}+e^{\phi}-\beta_{1} e^{\phi}+k e^{\phi}+\rho e^{\phi}-\rho \beta_{2} e^{\phi}\right. \\
& \left.-c c_{1} e^{\phi} \psi / \hat{T}_{s}^{2}\right), \\
s_{2}= & 4\left(c_{2} e^{\phi}-\beta_{1} c_{2} e^{\phi}+c_{2} k e^{\phi}+k e^{2 \phi}+c_{2} \rho e^{\phi}\right. \\
& -\beta_{2} c_{2} \rho e^{\phi}+\rho e^{2 \phi}-\rho \beta_{1} e^{\phi}-\rho \beta_{2} e^{2 \phi}+\rho k e^{2 \phi} \\
& \left.+c c_{1} k e^{2 \phi} / \hat{T}_{s}^{2} \ldots-c c_{1} e^{2 \phi} \psi / \hat{T}_{s}^{2}-c c_{1} \rho e^{2 \phi} \psi / \hat{T}_{s}^{2}\right), \\
s_{3}= & 2\left(c_{2} k e^{2 \phi}+c_{2} \rho e^{2 \phi}-\beta_{1} c_{2} \rho e^{2 \phi}-\beta_{2} c_{2} \rho e^{2 \phi}\right. \\
& +c_{2} \rho k e^{2 \phi}+\rho k e^{3 \phi}+c c_{1} k e^{3 \phi} / \hat{T}_{s}^{2} \\
& \left.+c c_{1} k \rho e^{3 \phi} / \hat{T}_{s}^{2} \ldots-c c_{1} \rho e^{3 \phi} \psi / \hat{T}_{s}^{2}\right) \\
s_{4}= & \left(c_{2} k \rho e^{3 \phi}+c c_{1} k \rho e^{4 \phi} / \hat{T}_{s}^{2}\right),
\end{aligned}
$$

The Routh-Hurwitz criterion is applied on above characteristic polynomial to obtain the governing algebraic equations for stability, namely, $s_{1} s_{2}-$

$$
J_{0}=\left[\begin{array}{l}
J_{11}, J_{12}, J_{13}, J_{14} \\
J_{21}, J_{22}, J_{23}, J_{24} \\
J_{31}, J_{32}, J_{33}, J_{34} \\
J_{41}, J_{42}, J_{43}, J_{44}
\end{array}\right]_{\left(\phi_{s s}, \psi_{s s}, \hat{\theta}_{2 s s}, \hat{\sigma}_{n s s}\right)}
$$

where abbreviations are defined as:

$$
\begin{aligned}
J_{11}= & -k e^{\phi_{s s}} / \hat{\sigma}_{n s s}-k e^{\phi_{s s}} \psi_{s s} \tan \alpha / \hat{\sigma}_{n s s}^{2} \\
& +\rho \beta_{2} e^{\phi_{s s}}+\left(\beta_{1}-1\right) e^{\phi_{s s}}, \\
J_{12}= & e^{\phi_{s s}} / \hat{\sigma}_{n s s}, \quad J_{13}=(\rho-1) \beta_{2} e^{\phi_{s s}}, \\
J_{14}= & -e^{\phi_{s s}} \psi_{s s} / \hat{\sigma}_{n s s}^{2}, \\
J_{21}= & -k e^{\phi_{s s}}, \quad J_{22}=0, \\
J_{23}= & 0, \quad J_{24}=0 \\
J_{31}= & -\rho e^{\phi_{s s}}, \quad J_{32}=0, \\
J_{33}= & -\rho e^{\phi_{s s}}, \quad J_{34}=0 \\
J_{41}= & k e^{\phi_{s s}} \tan \alpha, \quad J_{42}=0, \\
J_{43}= & 0, \quad J_{44}=0 .
\end{aligned}
$$

The characteristic equation is given $\left|J_{0}-\lambda I\right|=$ 0 , where $\lambda$ and $I$ are eigen value and identity matrix, respectively. The characteristic equation is expanded for getting the characteristic polynomial as $a_{0} \lambda^{4}+a_{1} \lambda^{3}+a_{2} \lambda^{2}+a_{3} \lambda+a_{4}=0$. The co-efficient of the polynomial is defined as the following

$$
a_{0}=16,
$$




$$
\begin{aligned}
a_{1}=8 & \left(e^{\phi}-\beta_{1} e^{\phi}+k e^{\phi} / \hat{\sigma}_{n}+\rho e^{\phi}\right. \\
& \left.-\beta_{2} \rho e^{\phi}+\psi e^{\phi} k \tan \alpha / \hat{\sigma}_{n}^{2}\right), \\
a_{2}= & 4\left(k e^{2 \phi} / \hat{\sigma}_{n}+\rho e^{2 \phi}-\beta_{1} \rho e^{2 \phi}\right. \\
& -\beta_{2} \rho e^{2 \phi}+k \rho e^{2 \phi} / \hat{\sigma}_{n}+k \psi e^{2 \phi} \tan \alpha / \hat{\sigma}_{n}^{2} \\
& \left.+k \psi \rho e^{2 \phi} \tan \alpha / \hat{\sigma}_{n}^{2}\right), \\
a_{3}=2 & \left(k \rho e^{3 \phi} / \hat{\sigma}_{n}+k \psi \rho e^{3 \phi} \tan \alpha / \hat{\sigma}_{n}^{2}\right), \\
a_{4}= & 0 .
\end{aligned}
$$

Finally, the Routh-Hurwitz criterion is used to obtain critical stiffness $k_{c r}$ at which sliding behaviour of the system changes from stable to unstable or vice versa. The Routh and Hurwitz criterion leads to the following system of coupled algebraic equations as $a_{1} a_{2}-a_{0} a_{3}=0$ and $a_{1} a_{2} a_{3}-a_{0} a_{3}^{2}-a_{4} a_{1}^{2}=0$. These equations are, in turn, solved numerically for critical stiffness $k_{c r}$ of the sliding system.

\section{References}

Becker T W 2000 Deterministic chaos in two state-variable friction slider and effect of elastic interaction; Geocomplex. Phys. Earthq. 1205.

Burridge R and Knopoff L 1967 Model and theoretical seismicity; Bull. Seismol. Soc. Am. 57(3) 341-371.

Brace W F and Byerlee J D 1966 Stick-slip as a mechanism of earthquake; Science 153 990-992.

Brittany A, Erickson B A, Birnir B and Lavallée D 2011 Periodicity, chaos and localization in a Burridge-Knopoff model of an earthquake with rate-and-state friction; Geophys. J. Int. 187(1) 178-198.

Carlson J M and Langer J S 1989 Properties of earthquake generated by fault dynamics; Phys. Rev. Lett. 62(22) 2632-2635.

Carlson J M and Langer J S 1989 Mechanical model of an earthquake fault; Phys. Rev. A. 40(11) 6470-6484.

Chau K T 1995 Landslides modelled as bifurcations of creeping slopes with nonlinear friction law; Int. J. Solid Struct. 32(23) 3451-3464.

Cochard A, Bureau L and Baumberger T 2003 Stabilization of frictional sliding by normal load modulation; $J$. Appl. Mech. 70(2) 220-226, https://doi.org/10.1115/1. 1546241.

Dieterich J D 1979 Modeling of rock friction: 1. Experimen results and constitutive equation; J. Geophys. Res. 84(B5) 2161-2168.

Dupont P E and Bapna D D 1994 Stability of the sliding frictional with varying normal force; J. Vib. Acoust. 116(2) $237-242$.

Faillettaz J, Sornette D and Funk M 2010 Gravitydriven instabilities: Interplay between state- and velocitydependent frictional sliding and stress corrosion damage cracking; J. Geophys. Res. 115 B03409, https://doi.org/ 10.1029/2009JB006512.

Gu Y and Wong T-F 1994 Nonlinear dynamics of the transition from stable sliding to cyclic stick-slip in rock in nonlinear dynamics and predictability of geophysical phenomena; In: Geophysical monograph (ed.) Newman W, AGU, Washington DC 8 15-35.

Gu J C, Rice J R, Ruina A L and Tse S T 1984 Slip motion and stability of a single degree of freedom elastic system with rate and state dependent friction; J. Mech. Phys. Solids 32 167-196.

Huang J and Turcotte D L 1990 Are earthquakes an example of deterministic chaos?; Geophys. Res. Lett. 17223.

Helmstetter A, Sornette D, Grassol J-R, Andersen J V, Gluzman S and Pisarenko V 2004 Slider-block friction model for landslides: Application to Vaiont and La Clapi'ere landslides; J. Geophys. Res. 109 B202409.

Kawamura H, Ueda Y, Kakui S, Morimoto S and Yamamoto T 2017 Statistical properties of the one-dimensional Burridge-Knopoff model of earthquakes obeying the rate- and state-dependent friction law; Phys. Rev. E 95 042122.

Linker M F and Dieterich J H 1992 Effect of variable normal stress on rock friction: Observation and constitutive equation; J. Geophys. Res. 97 4923-4940.

Lakshmanan M and Rajasekar S 2003 Nonlinear Dynamics (Integrability, Chaos and Patterns); Springer, Heidelberg.

Liu Y 2007 Physical basis of aseismic deformation transients in subduction zones; PhD thesis, Harvard University.

Marone C 1998 Laboratory-derive friction laws and their application to seismic faulting; Ann. Rev. Earth Planet. Sci. 26 643-696.

Niu Z-B and Chen D M 1994 Period-doubling bifurcation and chaotic phenomena in a single degree of freedom elastic system with a two-state variable friction law; In: Nonlinear Dynamics and Predictability of Geophysical Phenomena (eds) Newman W I, Gabrielov A and Turcotte D L, AGU, Washington DC, https://doi.org/10.1029/GM083p0075.

Niu Z B and Chen D M 1995 Lyapunov exponent and dimension of the strange attractor of elastic frictional system; Acta Seimol. Sinica 8 575-584.

Ranjith K and Rice J R 1999 Stability of quasi-static slip in a single degree of freedom elastic system with rate and state dependent friction; J. Mech. Phys. Solids 47 12071218.

Rice J R 1996 Slip complexity in dynamic model of earthquake faults; Proc. Nat. Acad. Sci. USA 93 38253829.

Rice J R and Ruina A L 1983 Stability of steady frictional slipping; J. Appl. Mech. 50 343-349.

Rice J R 1993 Spatio-temporal complexity of slip on a fault; J. Geophys. Res. 98 9885-9907.

Ruina A L 1983 Slip instability and state variable friction laws; J. Geophys. Res. 88 10359-10370.

Rice J R and Tse S T 1986 Dynamic motion of a single degree of freedom system following a rate and state dependent friction law; J. Geophys. Res. 91 521-530.

Segall P and Rice J R 2006 Does shear heating of pore fluid contribute to earthquake nucleation?; J. Geophys. Res. 111 B09316.

Shelly D R 2010 Periodic, chaotic, and doubled earthquake recurrence intervals on the deep san andreas fault; Science 238(11) 1385-1388.

Singh A K, Kanthola A and Singh T N 2012 Prediction of slope stability with an advanced friction model; Int. J. Rock. Mech. Min. Sci. 55 164-167. 
Singh A K and Singh T N 2013 Friction strength and steady relaxation using the rate and state dependent friction model; Pure Appl. Geophys. 170(3) 247-257.

Singh A K and Singh T N 2016 Stability of the rate, state, and temperature dependent friction model and its application; Geophys. J. Int. 205 636-647.

Sinha N and Singh A K 2016 Numerical study on chaotic behavior of slip and ageing laws of the rate and state friction; In: Dynamics, Vibration and Control, Narosa Publishing House, pp. 162-165.

Sinha N and Singh A K 2016 Linear and nonlinear stability analysis of rate state friction model with three state variables; Nonlin. Process Geophys. Discuss., https://doi. org/10.5194/npg-2016-11.

Strogatz S H 1994 Nonlinear dynamics and chaos: With application to physics, biology, chemistry and Engineering; Hachette, UK.

Tolstoi D M 1967 Significance of the normal degree of freedom and natural normal vibrations in contact friction; Wear 10 199-213.

Xuejun G 2013 Bifurcation behaviors of the two-state variable frictional law of a rock mass system; Int. J. Bifurcation Chaos. 23 11, https://doi.org/10.1142/ S0218127413501848.

Corresponding editor: N V CHALAPATHI RAO 